\title{
Molecular docking studies on SARS - Protease extracted from Wuhan COVID_19 strain by antiviral drugs
}

\section{Shivam Kumar}

Gautam Buddha university https://orcid.org/0000-0002-0966-1169

Aman Kumar ( $\nabla$ amanchahar123@gmail.com )

Gautam Buddha university

Keywords: PROTEASE , CHAIN A , USCF CHIMERA , RASMOL , Ganciclovir , SALBUTOMOL , PHENYLIC , CARBOXYL, BINDING AFFINITY .

Posted Date: May 3rd, 2020

DOI: https://doi.org/10.21203/rs.3.rs-26254/v1

License: (c) (i) This work is licensed under a Creative Commons Attribution 4.0 International License.

Read Full License 


\section{Abstract}

Background:

SARS - COVID 19 is a respiratory diseases in humans which is caused by the SARS - Coronavirus the treatment of coronavirus diseases- 19 has been continuously evolving and so far there has not been any Conesus treatment on an optimal level hasn't been evolved.

Motivation for this research:

The mainstream therapeutical involve broad spectrum antibiotic and antiviral drug that are already present are being used to treat the Pneumonia symptoms and effects and other drug called Hydroxychloro quinine is being used for fever symptoms and to prevent the binding of viral receptors to SARS - Protease enzyme present on COVID viral family to prevent further spread of viral symptoms and effect but this drug have side effect too like damaged liver, so in this research the aim is to test three mainstream antiviral drugs in molecular docking using first RASMOL to do the molecular study and then using USCF CHIMERA software to do the molecular modeling and to determine the best suited analog with very low binding energy to provide best results.

Also for treatment supportive immunity and core protein - ligand interaction plays very important part to determine structural based drug designing and simulation, in this present research three antiviral drugs namely Ganciclovir and SALBUTOMOL and their analog are being used and to study to determine least energy values which can predict the best suited drug for the treatment of effects and symptoms.

Several modifications were made to make drug compatible for simulation like addition of hydrogen, decreasing steric tension and addition of charges to non stranded groups modifications were made to Phenylic, Ketonic in case of Ganciclovir and Carboxyl group deletion in SALBUTOMOL and in USCF CHIMERA software.

Goals to be achieved:

Main objective of this research is to identify potential chemical compounds that can be docked in protease side chain of COVID 2 RNA genome which may act asa potential drug for clinical trial or possible treatment, two drugs SALBUTAMOL and GANCICLOVIR were docked .

Analogs were prepared by CHIMERA software and Protease from chain A of Corona RNA was selected for docking site in that stranded part and backbone was removed and just non stranded outer amino acid group were selected as active docking site for binding affınity.

Also in this research provides chances of further exploration was also provided for further exploration and observation based on drug binding affinity and energy values.

\section{Introduction}


Molecular docking is an attractive scaffold to understand drugbiomolecular interactions for the rational drug design and discovery, as well as in the mechanistic study by placing a molecule (ligand) into the preferred binding site of the target specific region of the DNA/protein (receptor) mainly in a non-covalent fashion to form a stable complex of potential efficacy and more specificity. The information obtained from the docking technique can be used to suggest the binding energy, free energy and stability of complexes. At present, docking technique is utilized to predict the tentative binding parameters of ligandreceptor complex beforehand.

The main objective of molecular docking is to attain ligand-receptor complex with optimized conformation and with the intention of possessing less binding free energy. The net predicted binding free energy $\left(\Delta G_{\text {bind }}\right)$ is revealed in terms of various parameters, hydrogen bond $\left(\Delta G_{h b o n d}\right)$, electrostatic

$\left(\Delta \mathrm{G}_{\text {elec }}\right)$, torsional free energy $\left(\Delta \mathrm{G}_{\text {tor }}\right)$, dispersion and repulsion $\left(\Delta \mathrm{G}_{\mathrm{vdw}}\right)$, desolvation $\left(\Delta \mathrm{G}_{\text {desolv }}\right)$, total internal energy $\left(\Delta \mathrm{G}_{\text {total }}\right)$ and unbound system's energy $\left(\Delta \mathrm{G}_{\mathrm{unb}}\right)$. Therefore, good understanding of the general ethics that govern predicted binding free energy $\left(\Delta \mathrm{G}_{\text {bind }}\right)$ provides additional clues about the nature of various kinds of interactions leading to the molecular docking .

non-covalent interactions in protein-ligand complexes is essential in modern biochemistry and should contribute toward the discovery of new drugs. The affinity of a ligand toward its receptor falls into a range of $10-80 \mathrm{~kJ} / \mathrm{mol}$. It is related to the binding constant and corresponds to a free energy. Accordingly enthalpic and entropic effects determine binding affinity. Hydrogen bonds and lipophilic contacts are the most important contributions to protein-ligand interactions. They are governed by changes in entropy and enthalpy. Solvation and desolvation effects either of the ligand and the protein binding site play a key role in the binding process. Prerequisite for a quantitative description and subsequently for a prediction of protein-ligand interactions is a partitioning in additive group contributions.

\section{Methods}

\section{Preparation and study of protein :}

Methods developed to facilitate and speedup the drug designing process are Rational Drug Design (RDD). These processes are used in biopharmaceutical industry to discover and develop new drugs. RDD uses a variety of computational methods to identify novel compounds. One of those methods is docking of drug molecules with receptors. The site of drug action, which is ultimately responsible for the pharmaceutical affect, is a receptor. SARS - Protease [Fig. 1] In order to find novel inhibitors, we computationally screened a compound library of over 687 million compounds for binding at the recently solved crystal structure of the main protease of SARS-CoV-2. A screening of such a vast chemical space for SARS-CoV2 protease inhibitors has not been reported before.

2. Collecting molecular structure

The collection of Ganciclovir and Salbutamol and SARS - Protease was identified via docking and their relative stabilities were evaluated using molecular dynamics and their binding affinities, using free energy 
simulations. There are various databases available, which offer information on small ligand molecules such as CSD (Cambridge Structural Database), ACD (Available Chemical Directory), MDDR (MDL Drug Data Report) and NCl (National Cancer Institute Database). Salbutamol is a short-acting, selective beta2adrenergic receptor agonist used in the treatment of asthma and COPD. It is 29 times more selective for beta 2 receptors than beta 1 receptors giving it higher specificity for pulmonary beta receptors versus beta1-adrenergic receptors located in the heart. Salbutamol is formulated as a racemic mixture of the Rand S-isomers. The R-isomer has 150 times greater affinity for the beta2-receptor than the S-isomer and the S-isomer has been associated with toxicity. This lead to the development of levalbuterol, the single Risomer of salbutamol. However, the high cost of levalbuterol compared to salbutamol has deterred widespread use of this enantiomerically pure version of the drug. Salbutamol is generally used for acute episodes of bronchospasm caused by bronchial asthma, chronic bronchitis and other chronic bronchopulmonary disorders such as chronic obstructive pulmonary disorder (COPD). It is also used prophylactically for exercise-induced asthma.

\section{Selection of ligand:}

In normal practice the conventional drugs like Ganciclovir , Salbutamol are used inhibit viral infection, which are specific to PROTEASE enzyme. Keeping rational drug designing approach in mind, We made an effort to design modified drugs for SARS, using conventional drugs like Ganciclovir and Salbutamol by docking against Protease receptor.

4. Selection of docking site:

The $\mathbf{C 3 0}$ Endopeptidase in various coronaviruses, often referred to as the $3 \mathrm{C}$-like proteinase ( $\left.3 \mathrm{CL}^{\text {pro }}\right)$, is a family of enzymes found in the Coronavirus polyprotein. It cleaves the polyprotein at two self-cleavage sites. It is a cysteine protease under clan PA, MEROPS classification C30. In coronaviruses, it is nonstructural protein number 5 (nsp5). The Enzyme Commission refers to this family as SARS coronavirus main protease.

\section{Molecular docking:}

Docking is the process of fitting together of two molecules in 3-dimensional space. Docking allows the scientist to virtually screen a database of compounds and predict the strongest binders based on various scoring functions. It explores ways in which two molecules, such as drugs and an enzyme SARS Protease fit together and dock to each other well, like pieces of a three-dimensional jigsaw puzzle. The molecules binding to a receptor, inhibit its function, and thus act as drug.

After shape screening, two docking protocols were applied followed by the determination of pharmacokinetically relevant molecular descriptors to narrow down the initial hits. Next, molecular dynamics simulations were conducted to validate the stability of docked binding modes and comprehensively quantify ligand binding energies. 


\section{Technical aspects:}

The simplest calculations can be performed by hand, but inevitably computers are required to perform molecular modeling of any reasonably sized system. The common feature of molecular modeling methods is the atomistic level description of the molecular systems.

The interactions between neighboring atoms are described by spring-like interactions (representing chemical bonds) and Van der Waals forces. The Lennard-Jones potential is commonly used to describe the latter. The electrostatic interactions are computed based on Coulomb's law. Atoms are assigned coordinates in Cartesian space or in internal coordinates, and can also be assigned velocities in dynamical simulations. The atomic velocities are related to the temperature of the system, a macroscopic quantity.

\section{TOOLS:}

Tools like CHIMERA and RASMOL and DISCOVERY STUDIO are very important for drug designing and simulation for possible targets and also to see the surface temperature gradient also the RASMOL which is used to make 3D mol modeling study which are explained below in two real time figures from RASMOL both in atom form and temperature map.

\section{PubMed:}

PubMed developed by the National Center for Biotechnology Information (NCBI) at the National Library of Medicine (NLM

\section{PDB:}

The structure of Angiotensin converting enzyme 2 (ACE-2) was retrieved from Protein data bank (PDB).

\section{RASMOL:}

The retrieved structure of COVID 2 Protease was analyzed by using RasMol.

\section{Discovery Studio:}

The structures of conventional drugs like Lopinavir and Ritonavir were retrieved from PDB and the structural analogs of these drug molecules were created by using Discovery Studio.

\section{Chimera molecular docking:}

Chimera software works on preparation of ligand and protein binding structures and also analyze tensions and hydrogen bonds, hydrophobic interactions, van der waal interaction, it also have tool auto dock vina for auto docking function . 


\section{Results}

Docking results between Protease and the conventional drug Salbutamol (Table I) as well as with the modified drugs are tabulated in Table 1.:

\begin{tabular}{|l|l|}
\hline ANALOG & E VLAUE \\
\hline ANALOG 1 & -2.60 \\
\hline ANALOG 2 & -2.60 \\
\hline ANALOG 3 & -2.50 \\
\hline ANALOG 4 & -2.50 \\
\hline ANALOG5 & -2.50 \\
\hline ANALOG 6 & -2.40 \\
\hline ANALOG7 & -2.30 \\
\hline
\end{tabular}

Based on Hydrogen bond value the number of hydrogen bond found in docking were 2 hydrogen bonds which is not good bonding sign.

While binding energy is good but there are not much hydrogen bondings and van der waal forces that increases the binding affinity and there are too much of steric forces that hinder in the binding of ligand to protein.

Table 2 : Docking results between Protease and the conventional drug Ganciclovir. (Table 2) as well as with the modified drugs are tabulated in Table 2:

\begin{tabular}{|c|c|}
\hline ANALOG & E VALUE \\
\hline ANALOG 1 & -2.6 \\
\hline ANALOG 2 & -2.6 \\
\hline ANALOG 3 & -2.5 \\
\hline ANALOG 4 & -2.5 \\
\hline ANALOG 5 & -2.4 \\
\hline ANALOG 6 & -2.4 \\
\hline ANALOG 7 & -2.3 \\
\hline ANALOG 8 & -2.3 \\
\hline
\end{tabular}


This is the best results with 61 hydrogen bonds and lowest energy and can work as potential ligand in this case.

\section{Conclusion}

The Protein-Ligand interaction plays a significant role in structural based drug designing. In the present work we have taken the receptor COVID 2 Protease enzyme 2and identified the drugs that can be used against this enzyme. They are Salbutamol and Ganciclovir. When the receptor (COVID Protease) was docked with two drugs the energy value obtained is: Ganciclovir. (-2.30), Salbutamol (-2.30). Further extension of this, We have tried to look for the most probable analog of the respective drugs, which specified earlier. When the modified drugs were docked against the same receptor the hydrogen bonds formation were different in each case and Ganciclovir was the perfect according to docking results which showed the formation of 61 hydrogen bonds and which shows the very strong interaction between ligand and protease chain which might be used in treatment for the for SARS owing to their high-energy value. This infers that the lead molecule is one with maximum interaction having high negative e-value. Thus the concept of protein-Ligand interaction helps in designing new drugs for SARS (Severe Acute Respiratory Syndrome). Further work can be carried out to improve the steric compatibility of the drugs based upon the work done above for a more energy efficient binding of the drug to the receptor.

In both cases it generated seven analogs all of them with different binding energies and lowest one were seventh and eighth in both cases but the hydrogen bond formation determine the strength of ligand binding and in cases of drug Ganciclovir it produced maximum amount of hydrogen bonds in seventh and eight analog form which were 61 hydrogen bonds which shows it's binding affinity as a ligand is very high.

\section{Declatations}

Competing interests: The authors declare no competing interests.

Funding: The authors received no external funding for this research project

\section{References}

1. http://en.wikipedia.org/wiki/Severe_acute_respiratory_syndrome\#Treatment

2. http://www.ncbi.nlm.nih.gov/PubMed.

3. http://www.pdb.org/.

4. Lee N, Allen Chan K, Hui D, Ng E, Wu A, Chiu R, Wong V, Chan P, Wong K and Wong E, "Effects of early corticosteroid treatment on plasma SARS-associated RNA concentrations in adult patients", Journal of Clinical Virology, 2004, 31(4): 304-309.

5. Murphy, Matthew. "Respiratory Pandemics of the 21st Century: Influenza A (H5N1) \& Severe Acute Respiratory Syndrome" [Internet]. Version 4. Knol. $2008 \mathrm{Jul}$ 
6. Stockman LJ, Bellamy R, Garner P. "SARS: systematic review of treatment effects". PLoS Med., 2006, 3 (9): 343.

7. Summary of probable SARS cases with onset of illness from 1 November 2002 to 31 July 2003". WHO. @ http://www.who.int/csr/sars/country/table2004_04_21/en/index.html.

8. Virupakshaiah DBM, Chandrakanth Kelmani, Rachanagouda Patil, and Prasad Hegade, "Computer Aided Docking Studies on Antiviral Drugs for SARS", World Academy of Science, Engg. and Tech., 2007,30

\section{Tables}

Table 1:

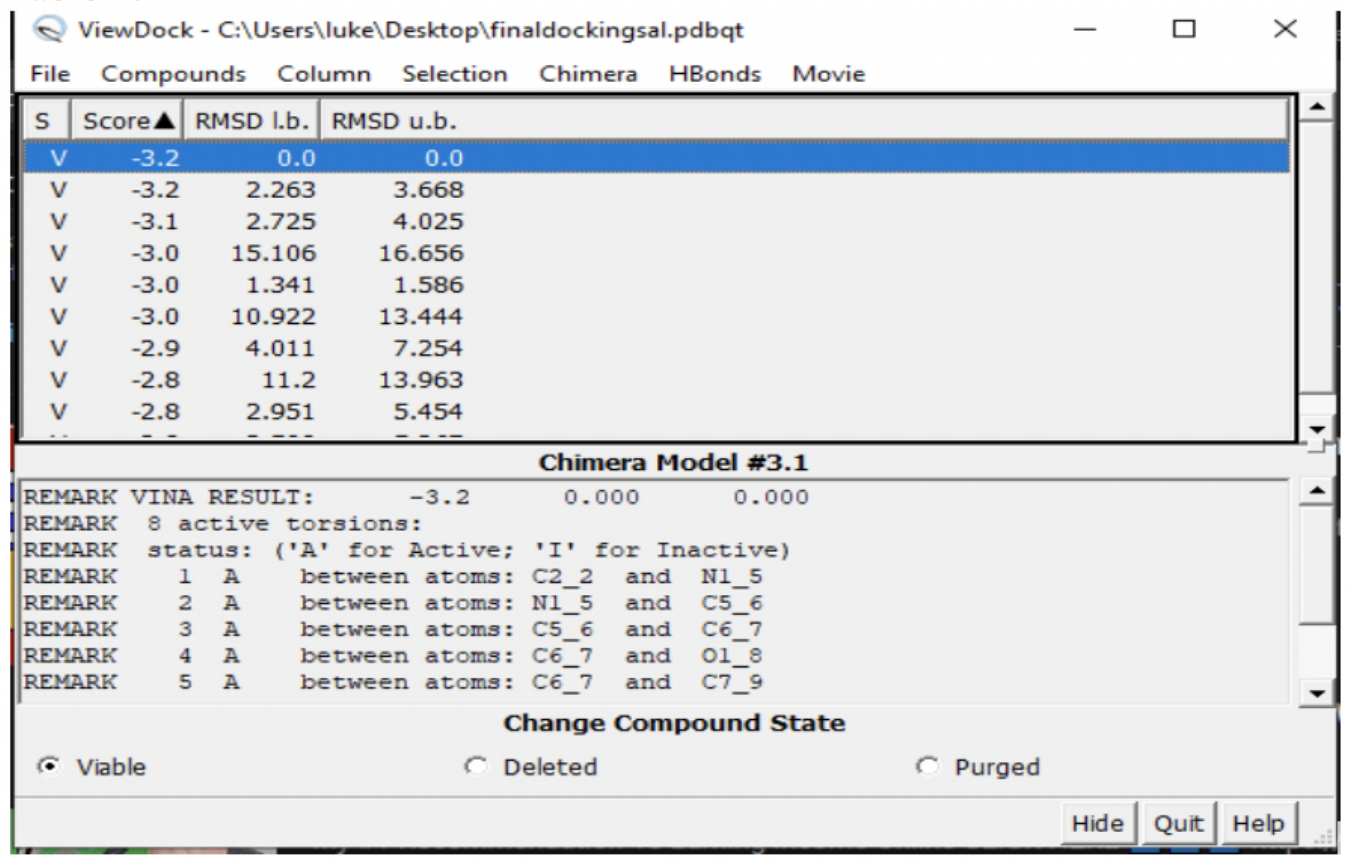


Table 2 :

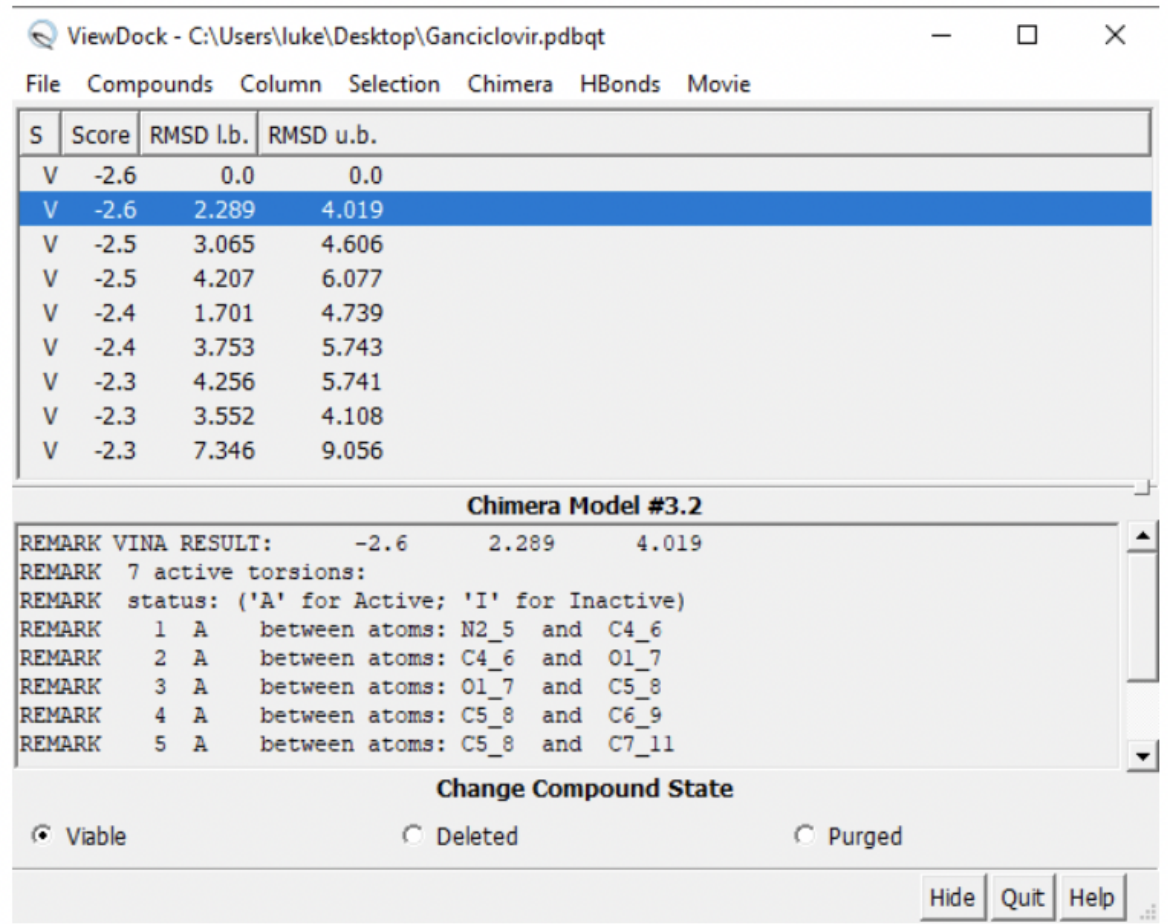

\section{Figures}

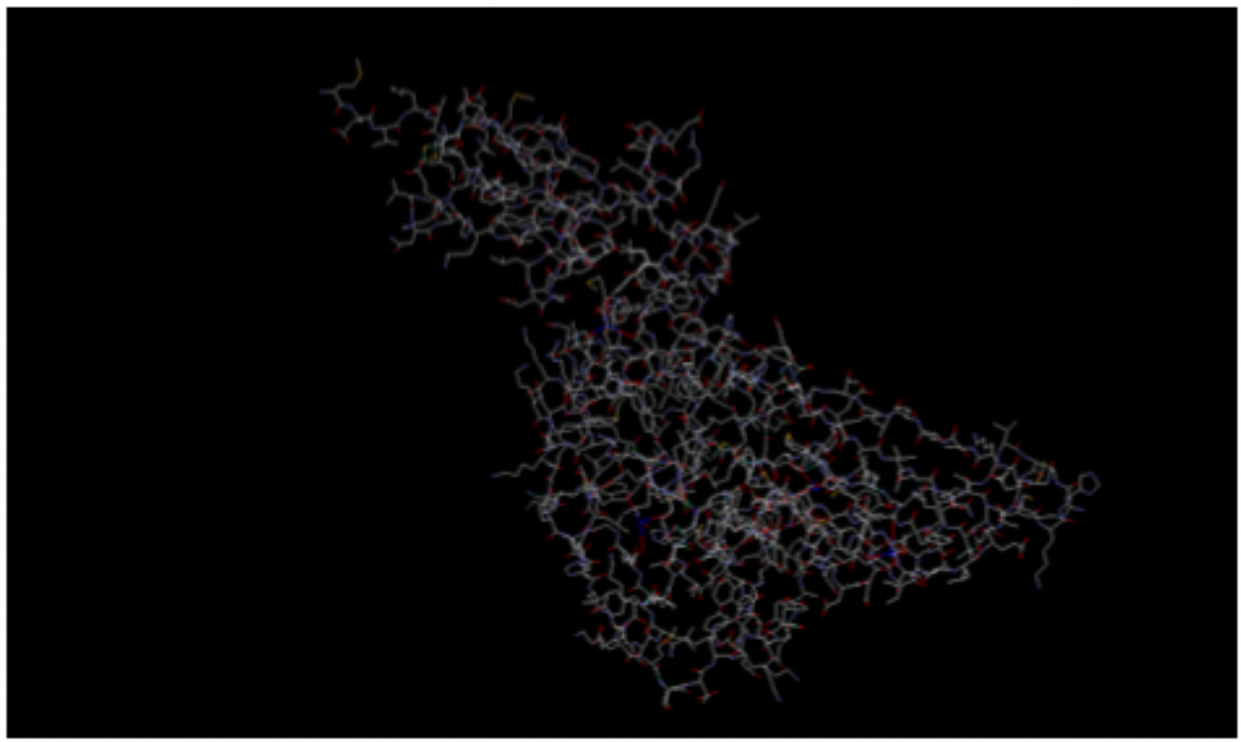

Figure 1

SARS PROTEASE 


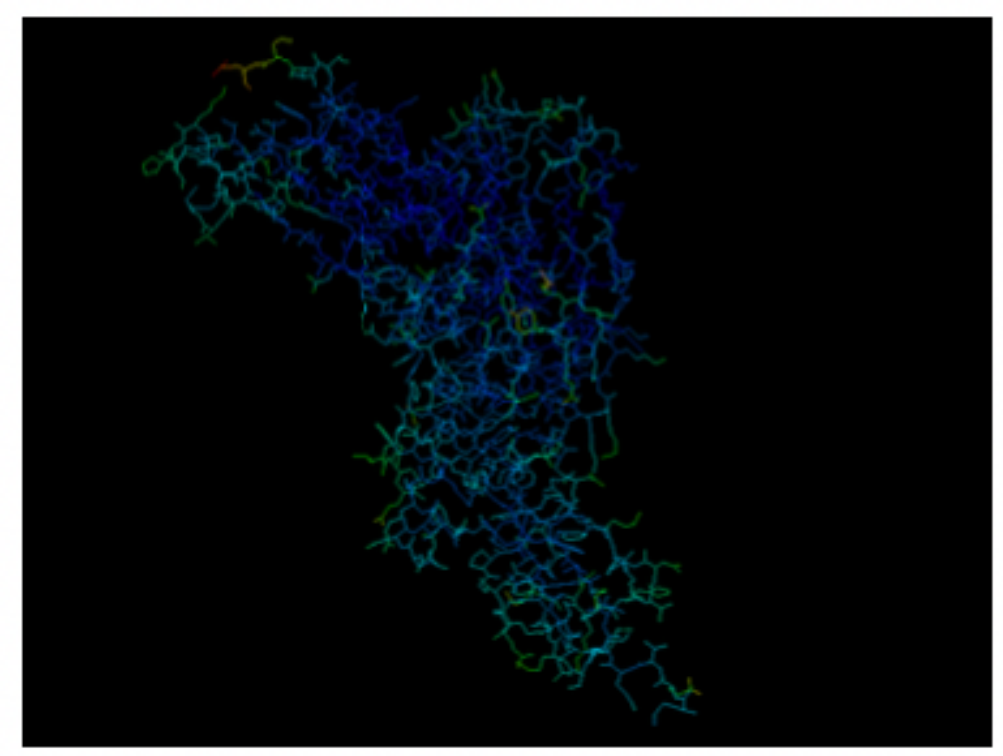

Figure 2

THERMAL MAP

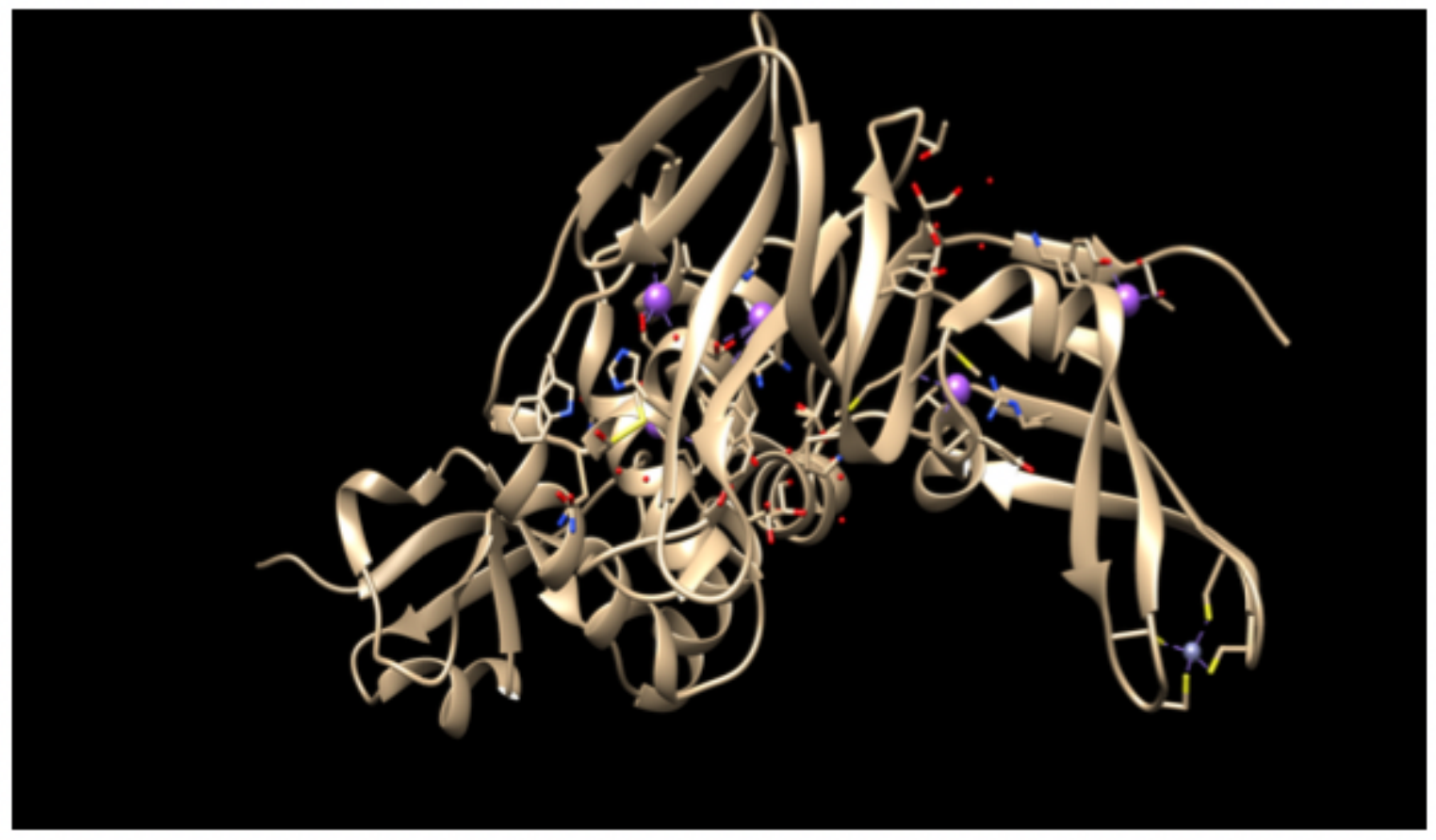

Figure 3

Below is the Protease structure extracted from SARS COVID - 19 in side chain A of Coronavirus RNA genome which have both stranded and non stranded residues and only non residual amino acids were used in docking. 


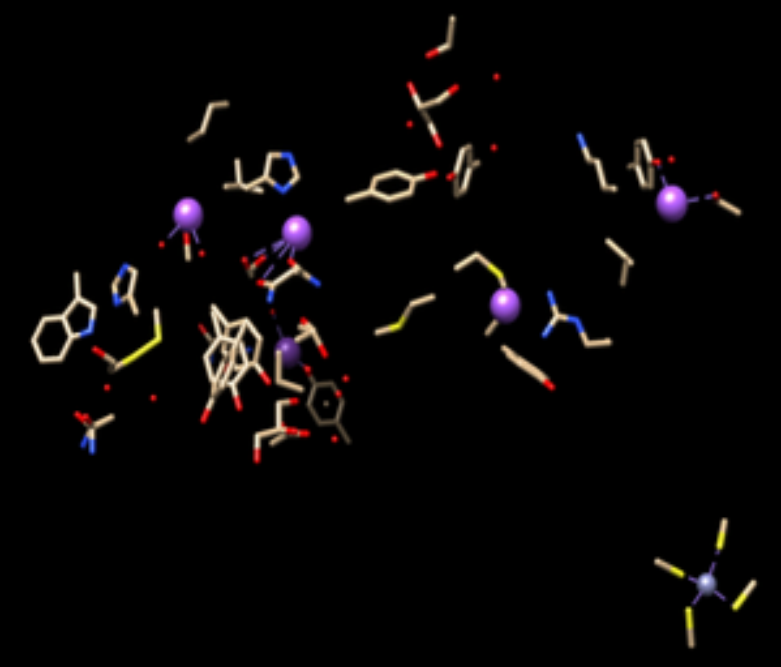

Figure 4

Below is the figure of non residual amino acids after the removal of residual component that may hinder in binding.

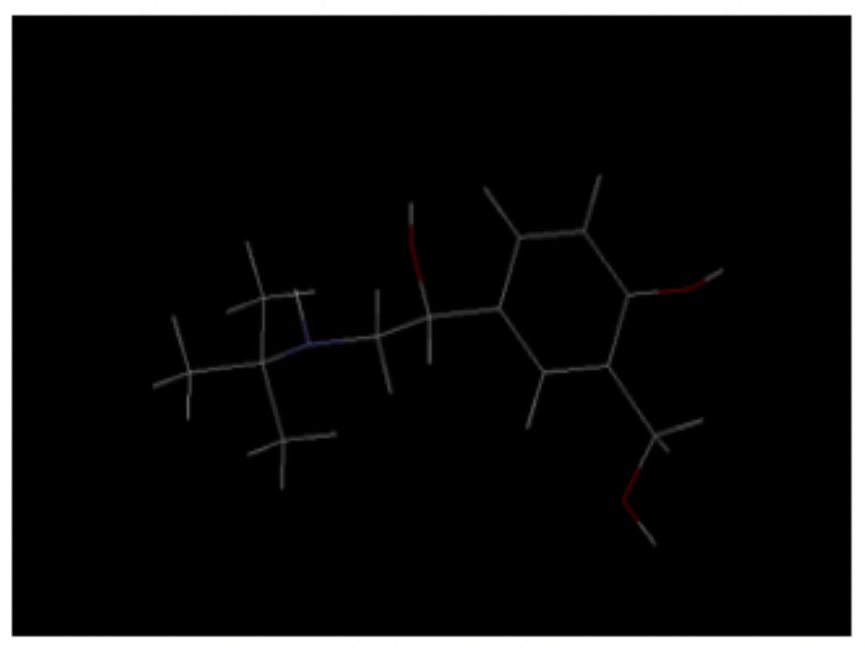

Figure 5

Ganciclovir. 


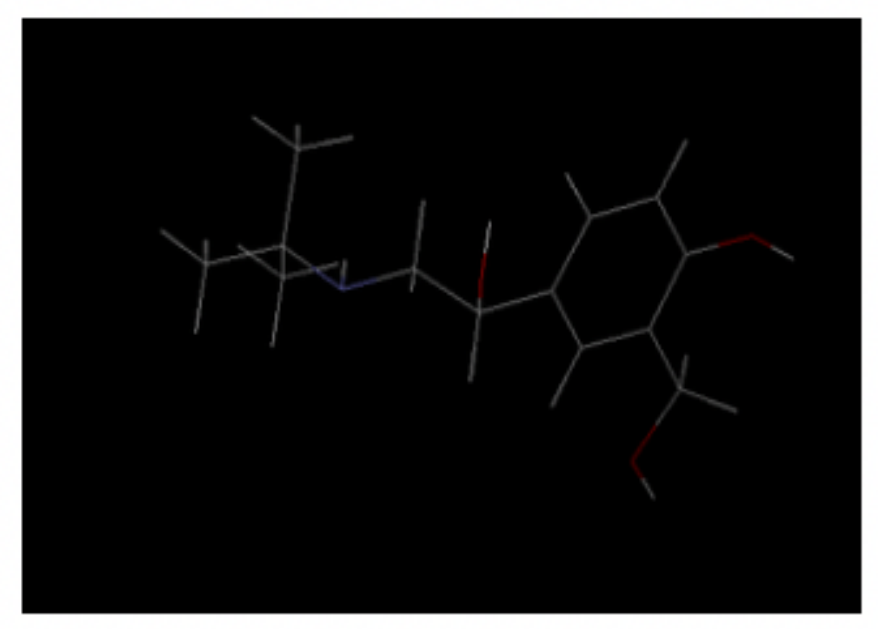

Figure 6

SALBUTAMOL.

\section{Supplementary Files}

This is a list of supplementary files associated with this preprint. Click to download.

- 5y3q.pdb 